\title{
PROCESS AND SYSTEMS Research into ageing and frailty
}

\author{
Authors: Adam L Gordon, ${ }^{A}$ Miles D Witham, ${ }^{B}$ Emily ] Henderson, ${ }^{C}$ Rowan H Harwood ${ }^{D}$ and Tahir Masud ${ }^{E}$
}

Research into ageing covers opportunities and challenges posed by an older population, and research to understand the ageing processes across the lifespan. The evidence base for Comprehensive Geriatric Assessment (CGA) is well established and efforts should now shift to understanding how to implement its principles across different healthcare contexts. Research around syndromes common in older people has progressed with variable success; while effective therapies for falls and cognitive impairment have been identified, older people with advanced frailty have commonly been excluded from Parkinson's disease and continence research. Research to understand the mechanisms of ageing has potential to mitigate against or treat emerging sarcopenia and cognitive impairment, and thus modify frailty trajectories. Pharmacogenetics could individualise therapeutics to reduce polypharmacy. These issues can only be addressed with development of infrastructure, capacity and expertise in ageing research. Commonly used research methodologies must be adapted to take account of frailty, cognitive impairment and functional dependency.

KEYWORDS: ageing, frailty, sarcopenia, cognitive impairment, research

DOI: $10.7861 /$ fhj.2021-0088

Authors: ${ }^{\text {A }}$ rofessor of the care of older people, British Geriatrics Society, London, UK, University of Nottingham School of Medicine, Nottingham, UK, NIHR Applied Research Collaboration-East Midlands (ARC-EM), Nottingham, UK and University of Hospitals of Derby and Burton NHS Foundation Trust, Derby, UK; ${ }^{\text {B }}$ professor of trials for older people, British Geriatrics Society, London, UK, NIHR Newcastle Biomedical Research Centre, Newcastle upon Tyne, UK and The Newcastle upon Tyne Hospitals NHS Foundation Trust, Newcastle-upon-Tyne, UK; ' Consultant senior lecturer in geriatric medicine, British Geriatrics Society, London, UK, Bristol Medical School, Bristol, UK and Royal United Hospitals Bath NHS Foundation Trust, Bath, UK; D professor of palliative and endof-life care, British Geriatrics Society, London, UK, University of Nottingham School of Health Sciences, Nottingham, UK and Nottingham University Hospitals NHS Trust, Nottingham, UK; Eprofessor of geriatric medicine, British Geriatrics Society, London, UK, University of Nottingham School of Medicine, Nottingham, UK, Nottingham University Hospitals NHS Trust, Nottingham, UK and NIHR Nottingham Biomedical Research Centre, Nottingham, UK

\section{Introduction}

Widespread population ageing is a global phenomenon driven by changes in life expectancy and declining fertility. Although historically a problem of higher income countries, it now affects all parts of the world, with the most rapid population ageing now occurring in lower- and middle-income countries (LMICs). ${ }^{1}$ By 2050 , the number of people aged 60 years or over in LMICs is expected to increase more than two-fold, from 652 million to 1.7 billion, whereas higher income countries are projected to see a $38 \%$ increase over that period. $^{2}$

Lay media commentaries tend to catastrophise global ageing as a 'silver tsunami', where the increased number of older people is seen as contributing to increasing dependency ratios and an epidemic of frailty that could overwhelm social security, and health and social care systems. ${ }^{3}$ There is evidence that economic and care system reform will be necessary to meet the needs of an ageing population, but there is also an important counternarrative about the role that older people can and should play in society by remaining economically active for longer, volunteering or caring for family members. ${ }^{4,5}$

The research agenda around ageing and frailty could be seen as having two main components: understanding and responding to opportunities and challenges posed by an aged population, and understanding the ageing process to maximise positive aspects and mitigate negative impacts. Research into ageing has historically faced significant underinvestment by comparison with other fields, and attention must be paid to developing the infrastructure required to support research under both these headings if we're to make global ageing a human success story. ${ }^{6}$

Understanding and responding to opportunities and challenges posed by an aged population

Clinicians most often approach ageing from the perspective of increasing prevalence of frailty in clinical populations and how this influences clinical care. Emergent frailty in older populations shaped the origin story for geriatric medicine and has continued to influence its development and delivery since. ${ }^{7,8}$ Initial "blackbox' randomised controlled trials (RCTs) to build an evidence base for Comprehensive Geriatric Assessment (CGA) have been followed by serial meta-analyses, qualitative studies and reviews to understand its 'active ingredients'. ${ }^{9-15}$ More focused studies have gone on to evaluate variants of CGA after traumatic fracture, perioperatively, in those with delirium and dementia, and in the context of Hospital at Home. ${ }^{16-19}$

Further trials of CGA are almost certainly only justified where the clinical population is different in some way from those in 
which it has already been shown to work. Re-evaluating CGA in every clinical context where older people appear is unlikely to be an effective use of resource. There remain, however, important questions about implementation. ${ }^{20}$ Recent work looking at implementation of CGA in UK care homes found the language and conceptual framework of CGA to be alienating for some community-based practitioners, not least because some of the 'active ingredients' in CGA are already present and widely deployed in community settings. ${ }^{21}$ There is similar overlap with principles of good palliative care. ${ }^{22}$ Adherence to a strict mantra of CGA as a 'plug and play' intervention therefore could stifle evidence-based care of older people in some settings. Research-based approaches to understanding the context of implementation (such as the Consolidated Framework for Implementation Research (CFIR) or Promoting Action on Research Implementation in Health Services (PARIHS) frameworks) have much to offer, as do more nuanced approaches to interpreting the real-world application of evidence, such as realist synthesis and evaluation (describing what works, for whom and under what circumstances). ${ }^{23-26}$

This phase-shift to implementation science in CGA, rather than simply testing its effectiveness over and over, might be seen as news by some geriatricians but, in reality, many of the most important gains in care of older people have been made by the context-sensitive implementation of evidence-based approaches developed by other disciplines. The impact of cataract surgery on mobility and falls, improved outcomes following hip replacement in even the most disabled patients, and extension of cancer therapies into older populations have all been characterised by adaptation and modification of existing approaches, with attention to quantifying clinical benefit in older populations with or without recourse to RCTs as needed. ${ }^{27-30}$ Continually appraising the medical literature for emerging therapies that may require modification to work in older people, some of whom are frail, then choosing the appropriate research approach to evidence and support clinical impact, is likely to remain an important component of research into ageing.

The common presenting features of illness in older adults and, therefore, foundation of geriatric medicine were memorably described by Bernard Isaacs as 'geriatric giants': cognitive impairment, incontinence, immobility and instability. ${ }^{31}$ Research into care for older people continues to be defined in some parts by ongoing research into syndromes common in later life. Clinical research into falls and bone health interventions has been particularly successful, with evidence that multifactorial interventions can reduce falls and fracture risk. ${ }^{32}$ This part of the ageing research agenda has now matured to the point where questions largely relate to nuances about how to implement evidence-based approaches, or how to adapt them in light of emerging technologies or new evidence from basic science (subtending the sarcopenia agenda discussed later). ${ }^{33}$ Pharmacological treatments for those with established dementia or cognitive impairment have been the focus of considerable research effort but have hitherto not demonstrated significant disease-modifying effects, although they have shown some impact on cognitive and non-cognitive symptoms, and carer burden. ${ }^{34,35}$ Research into stopping or minimising antipsychotic prescribing in the context of dementia, most of which has shown little or no harm, has encouraged much more critical and cautious approaches to prescribing such drugs. ${ }^{36}$ Non-pharmacological interventions in dementia continue to be advocated on the basis of clinical experience and the recognition that pharmacological approaches offer little but the evidence base for such interventions is underdeveloped and principles of experimental psychology have much to offer here. ${ }^{37,38}$ By systematising and understanding what works, and when, in dementia care, it's likely that ever more individualised, nuanced and effective care can be delivered in a more systematic way. Cohort and interventional studies in other neurodegenerative diseases (such as Parkinson's disease) have tended to exclude older people by design. ${ }^{39}$ Failure to consider comorbid conditions, the physical demands of multiple site visits and the impact of lengthy outcome assessment measurements have limited the feasibility of such work in those with significant frailty. Disease-related fatigue, apathy, cognitive impairment, motor fluctuations and the regular necessity for 'off' medication state assessments all need to be better integrated into the fabric of trial design if the most vulnerable people with Parkinson's disease are to be included in research. Finally, continence research in older people remains a largely underexplored area, with faecal incontinence being particularly affected by a paucity of evidence. $^{40}$

Age is, at best, a proxy for clinical vulnerability and risk, as any geriatrician faced by the question, 'How old do you need to be to see a geriatrician?' will tell you. The emergence of frailty as measurable phenomenon (whether as the phenotypic outcome of a cascade of homeostatic decompensation or as a more straightforward count of accumulated deficits) has provided a more robust way to categorise populations according to risk. ${ }^{41,42}$ The emergence of electronic versions of frailty indices that can be embedded in healthcare databases provides the opportunity for big data approaches that can enable us to better understand how older people interact with, and benefit from, health and social care in later life. ${ }^{43}$ This will underpin the implementation agenda as described earlier. Frailty better approximates clinical vulnerability than age, but it remains a syndromic description of multi-system decompensation, and thus an imperfect expression of its underlying components. ${ }^{44}$ It may, over time, become less relevant as we understand in greater detail, and are better able to measure, how function declines across multiple body systems as we age.

\section{Research into ageing}

Understanding the fundamental biological mechanisms that underpin the ageing process, provides opportunities to maximise independence and participation, and minimise disability. Lifecourse perspectives of ageing (that decisions and events occurring throughout the lifespan influence ageing processes) have long held sway in the social sciences. ${ }^{45}$ Increasingly, data from a range of biomedical studies have reinforced this. Epigenetic studies have highlighted how maternal decisions influence successful ageing in offspring, while longitudinal cohort studies have outlined the impact of adolescent and mid-life exposures on ageing. ${ }^{46,47}$

Research that identifies why, how and when changes begin that later go on to define the frailty state is now a key focus of research into ageing, with particular focus on key ageing syndromes, for example, sarcopenia and cognitive impairment.

Sarcopenia refers to the age-related loss of muscle strength and mass seen with advancing age. ${ }^{48}$ Although recognised for centuries, it has been operationalised as a diagnosis only over the last decade or two. Sarcopenia is common, with $5 \%-10 \%$ of over 65-year-olds affected and with much higher rates in care home and hospital populations. ${ }^{49}$ Its critical importance is as a 
key component of the physical frailty syndrome, and as a major contributor to fall risk, increased length of hospital stay, need for care and earlier death. ${ }^{50}$ As with many ageing syndromes, the aetiology is complex and multifactorial. Epidemiological studies have shown strong associations between multiple long-term conditions and sarcopenia. ${ }^{51}$ Advances in discovery science have highlighted a range of fundamental biological processes implicated in sarcopenia including inflammation, cellular senescence, mitochondrial dysfunction, neuromuscular junction dysregulation, metabolic dysregulation and disrupted paracrine and endocrine signalling. ${ }^{52,53}$ These advances provide multiple targets for nutritional and pharmacological candidate interventions, either to improve sarcopenia directly or to augment the effect of resistance exercise training, which is the only intervention shown unequivocally to improve muscle strength and mass in older people with sarcopenia. ${ }^{49}$

Cognitive impairment and dementia are the end stage of a series of pathophysiological changes that begin in the brain in early life. The cellular and end-organ changes that lead in a stepwise fashion to each of the common dementia syndromes (Alzheimer's dementia, vascular dementia, Lewy-body disease and frontotemporal dementia) are increasingly well understood and are being investigated as putative targets for pharmacological and non-pharmacological interventions. ${ }^{37,54,55}$ Epidemiological studies suggest that many of the changes associated with increased risk of dementia correlate with exposures and accumulated deficits in early or later life: hypertension, hearing impairment, smoking, obesity, depression, physical inactivity, diabetes, social isolation, poor education, alcohol consumption, traumatic brain injury and air pollution. ${ }^{37}$ Drugs with anticholinergic burden are likely to fuel underlying neurochemical deficiencies and negatively impact cognitive outcomes. ${ }^{56}$ Identifying these associations enables researchers to focus on interventions that could either modify exposures or direct pharmacological strategies towards pathophysiological mechanisms which mediate cellular damage and cognitive decline.

Progress in understanding the physiological and pathophysiological processes underpinning ageing, with sarcopenia and cognitive decline being important examples, has opened up a field of interest around senolytic and senostatic drugs, which are viewed as having potential to abort or slow the ageing process, respectively. Several of these medications, which target one or more molecular target associated with cellular ageing, have shown efficacy in preclinical trials. Human studies are currently evaluating their impact on long-term conditions and also how ageing attenuates response to acute illnesses including infection. ${ }^{57,58}$

Understanding of fundamental biology and the aetiology of ageing, and the way in which this influences vulnerability to disease, is also the most likely route to improve our understanding and management of multiple long-term conditions (MLTC). MLTC (defined as two or more chronic medical conditions) affect the majority of older people, and pose major challenges of symptom and treatment burden. ${ }^{59}$ Their presence drives polypharmacy with its attendant side effects and prescribing cascades, and both outcomes and satisfaction with healthcare are low for people living with MLTC. ${ }^{60}$ The current chief medical officer of England highlights MLTC as one of the great challenges that health systems are failing to meet. ${ }^{61}$

One area of promise with regard to combatting polypharmacy is the field of pharmacogenetics. The use of pharmacogenomic testing as a decision support tool could potentially identify individual patients at risk of medication-related adverse effects, inform dosing and increase the chance of treatment response. This could help with medication selection and help with patient outcomes, although further research is needed to understand how best to implement such testing into clinical contexts. ${ }^{62}$ As an example, emerging data suggest that genetic variation in cytochrome P450 enzymes contributes to heterogeneity in the clinical effectiveness and side-effects of antidepressants. ${ }^{63}$ Further developments in pharmacogenomics could enable a more individualised approach to older people, maximising efficacy and minimising medication-related adverse effects.

\section{Developing capacity in ageing research}

Ageing challenges methods widely deployed in clinical research. ${ }^{64}$ Cognitive impairment and physical frailty influence both concordance and response rates, and introduce floor and ceiling effects on outcome measures that are widely deployed in younger populations. MLTC introduce confounding so that relationships between interventions and outcomes need more careful unpicking. Studies in long-term care facilities can be impacted by cross-contamination, which mean that cluster randomisation is required. ${ }^{65}$ Long-term care also often operates in the grey space between social care and healthcare and this can challenge both research governance and enlisting the support of the relevant professional constituencies. ${ }^{66}$ The vulnerability of many older people means that the case for research equipoise needs to be carefully made if investigations are to be ethically tenable. All of these issues require investment in people to develop expertise in designing and delivering research for older people, and a number of initiatives are underway to train the next generation of clinical academics and local investigators across all professions to support research for older people. ${ }^{6,67}$ Research infrastructure including National Institute for Health Research (NIHR) Biomedical Research Centres (the Newcastle centre specialises in ageing research), the NIHR Applied Research Collaboratives and the NIHR Ageing Clinical Research Network are key to building this training capacity. The NIHR School for Social Care Research has a remit around building capacity in social care research. A key goal from NIHR must be integrating the research outputs from its social and healthcare workstreams and that these don't simply take place in parallel.

Historically, the difficulty of recruiting older people meant that enrolling representative older populations was avoided and put in the 'too difficult' pile. ${ }^{64}$ This continues to be the case in some instances; for example, although SARS-COV-2 vaccination studies were sometimes conducted in older cohorts, they did not include older people with MLTC and advanced frailty who were most likely to be adversely affected by COVID-19. ${ }^{68}$ The ensuing debates in some countries about whether older people could benefit from vaccination and even whether it was ethical to administer it to them were a stark reminder of how a lack of data can reinforce ageist tropes. ${ }^{69}$ Emerging evidence from countries where vaccination in such groups was prioritised have demonstrated effectiveness. ${ }^{70}$ Future exclusion of older people with frailty and multimorbidity from clinical trials will further reinforce structural ageism and result in them being denied treatments from which they could stand to benefit. In parallel, while excluded from trial participation, they remain exposed to relatively unquantified harms from interventions being delivered 
Fig 1. Roadmap to improve inclusion of under-represented groups, including older people, in clinical research. Reproduced with permission from Witham MD, Anderson E, Carroll C et al. Developing a roadmap to improve trial delivery for under-served groups: Results from a UK multi-stakeholder process. Trials 2020;21:694.

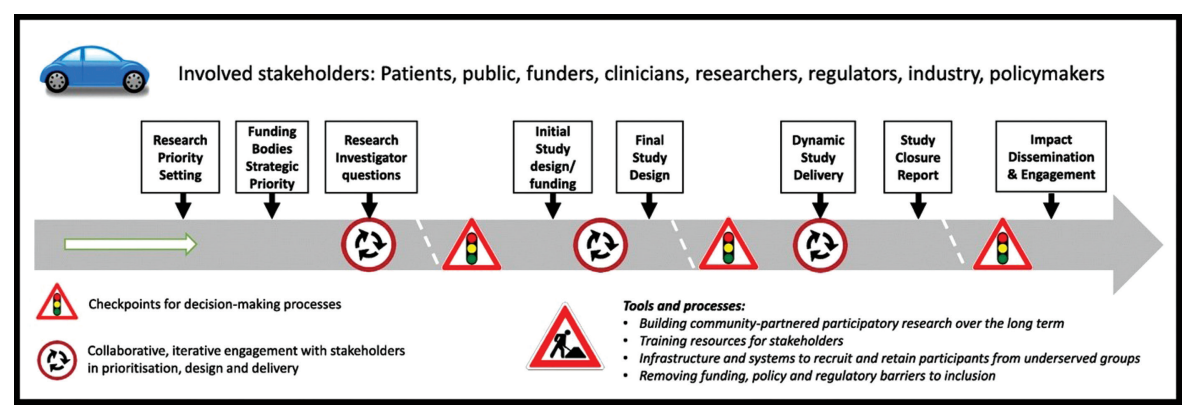

on the basis of extrapolated evidence of efficacy in younger, more robust and physiological resilient populations. The NIHR INCLUDE project has catalysed work to improve inclusion of under-served groups (including older people, those living with MLTC, those with cognitive impairment and who live in care homes) into clinical research through a series of guidelines, training initiatives, engagement with regulators and funders, and context-specific tools to help researchers design and deliver more inclusive research (Fig 1). ${ }^{71}$ For older people, this requires embracing the complexity that accompanies MLTC, with pragmatic inclusion and exclusion criteria. Equally important is fitting the research to the participant, not the participant to the research. Suitable approaches include provision of transport, conducting visits in participants' homes, simplifying and enlarging information sheets, reducing the length and burden of outcomes visits, recognising that older ethnic minority populations do not always have English as a first language, and enabling participation for those unable to consent for themselves. ${ }^{72}$

Considered recruitment strategies are essential if the conclusions drawn from research are to be meaningful to the population who are living with the condition under study. In fundamental research into ageing, the physiology and pathology of becoming older cannot be understood by focusing on healthy volunteers alone. An example is how muscle mass and quality indices developed in heathy volunteers can have limited utility in clinical populations which are similar in chronological age, because of markedly diminished muscle mass in clinical cohorts. ${ }^{73}$ Clinical trials for hypertension offer an example where there has been a focus on recruiting healthy older people with consequent lack of generalisability to clinical cohorts. Even when the mean age in trials is high, the populations studied are much healthier than typical patients seen in clinic. The results, therefore, do not tell us anything about the balance between benefit and risk of antihypertensives in older people living with frailty or dementia; the groups where therapeutic uncertainty is highest. ${ }^{74}$

The technical expertise to deliver studies for older people is developing apace. A useful example of how these can be usefully deployed comes from the PROTECT-CH platform trial for prophylaxis of COVID-19 in care homes for older people. ${ }^{75}$ The suite of measures adopted includes use of linked routine datasets to record outcome measures and minimise data burden on participants; remote video-consenting to maximise access to consultees to optimise inclusion of those who lack capacity to consent; use of validated proxy measures for subjective outcomes such as quality of life in those with dementia; use of cluster randomisation to account for contamination within treatment arms; and a pragmatic approach to adherence and outcome measurement designed to accommodate the limited capacity of care home residents to follow protocols. PROTECT-CH is designed as a potential future platform for RCTs of investigational medicinal products (IMPs) in older people with frailty who live in care homes. As such, it has had to develop approaches to consent, research governance and indemnity in the context of IMP trials for the first time in UK care homes. This highlights the importance of investing in such infrastructure projects to build the necessary technical and governance expertise to overcome the complexity of trials in older people.

Novel and innovative application of trial methodologies may be complemented by improved collection and collation of real-world clinical data which, coupled to artificial intelligence and machine learning approaches, could shed new light on associations between exposures and outcomes. The equipoise around management of hypertension in older people with advanced frailty, as described earlier, is one area where such approaches have promise. $^{76}$

\section{Conclusion}

Delivering research evidence that is relevant to the needs of older people and the health and social care systems that care for them requires a full-spectrum approach, from discovery science to implementation. At present, the quantity of research focused on the needs of older people is not commensurate with either clinical need or clinical service delivery. However, recent advances in the biology of ageing, and investment in building the infrastructure and people needed to design and deliver research for older people mean that the stage is set for rapid growth in translational and applied ageing research. These advances, together with a growing realisation by funders and policymakers that ageing research is critical to the future health and wealth of countries, now need to be combined to transform health and care for all of us as we age. ${ }^{77}$.

\section{References}

1 World Health Organization. Ageing and health: key facts. WHO, 2018. www.who.int/news-room/fact-sheets/detail/ageing-andhealth [Accessed 30 April 2021].

2 United Nations. World population ageing. UN, 2017. www.un.org/ en/development/desa/population/publications/pdf/ageing/ WPA2017_Highlights.pdf [Accessed 30th April 2021].

3 Calasanti T. Brown slime, the silver tsunami, and apocalyptic demography: The importance of ageism and age relations. Social Currents 2020;7:195-211. 
4 Government Office for Science. Future of an ageing population. Government Office for Science, 2016. https://assets.publishing. service.gov.uk/government/uploads/system/uploads/attachment_ data/file/816458/future-of-an-ageing-population.pdf [Accessed April 30 2021].

5 Zaidi A, Gasior K, Zolyomi E et al. Measuring active and healthy ageing in Europe. Journal of European Social Policy 2017;27:138-57.

6 Witham MD, Roberts HC, Gladman ] et al. Growing research in geriatric medicine. Age Ageing 2019;48:316-9.

7 Barton A, Mulley G. History of the development of geriatric medicine in the UK. Postgrad Med J 2003;79:229-34.

8 Morley JE. A brief history of geriatrics. J Gerontol A Biol Sci Med Sci 2004;59:1132-52.

9 Stuck AE, Siu AL, Wieland GD et al. Comprehensive geriatric assessment: a meta-analysis of controlled trials. Lancet 1993;342:1032-6.

10 Ellis G, Gardner M, Tsiachristas A et al. Comprehensive geriatric assessment for older adults admitted to hospital. Cochrane Database Syst Rev 2017;9:CD006211.

11 Ellis G, Whitehead Martin A, O'Neill D et al. Comprehensive geriatric assessment for older adults admitted to hospital. Cochrane Database Syst Rev 2011:CD006211.

12 Ellis G, Langhorne P. Comprehensive geriatric assessment for older hospital patients. Br Med Bull 2005;71:45-59.

13 Chadborn NH, Goodman C, Zubair M et al. Role of comprehensive geriatric assessment in healthcare of older people in UK care homes: realist review. BMJ Open 2019;9:e026921.

14 Reuben DB, Fishman LK, McNabney M et al. Looking inside the black box of comprehensive geriatric assessment: a classification system for problems, recommendations, and implementation strategies. J Am Geriatr Soc 1996;44:835-8.

15 Alibhai SMH, Jin R, Loucks A et al. Beyond the black box of geriatric assessment: Understanding enhancements to care by the geriatric oncology clinic. J Geriatr Oncol 2018;9:679-82.

16 Eamer G, Taheri A, Chen SS et al. Comprehensive geriatric assessment for older people admitted to a surgical service. Cochrane Database Syst Rev 2018;1:CD012485

17 Sbai M, Martin F, Partridge ] et al. Comprehensive geriatric assessment (CGA) in the perioperative setting: The current state of play. J $R$ Coll Physicians Edinb 2020;50:356-8.

18 Goldberg SE, Bradshaw LE, Kearney FC et al. Care in specialist medical and mental health unit compared with standard care for older people with cognitive impairment admitted to general hospital: Randomised controlled trial (NIHR TEAM trial). BMJ 2013;347:f4132.

19 Shepperd S, Butler C, Cradduck-Bamford A et al. Is comprehensive geriatric assessment admission avoidance hospital at home an alternative to hospital admission for older persons? Ann Intern Med 2021 [Epub ahead of print]

20 Gladman JRF, Conroy SP, Ranhoff AH et al. New horizons in the implementation and research of comprehensive geriatric assessment: knowing, doing and the "know-do" gap. Age Ageing 2016:45:194-200.

21 Devi R, Chadborn NH, Meyer ] et al. How quality improvement collaboratives work to improve healthcare in care homes: a realist evaluation. Age Ageing 2021; afab007 [Epub ahead of print].

22 Enguell $\mathrm{H}$, Harwood $\mathrm{RH}$. What palliative care can learn from geriatric medicine. Br J Hosp Med (Lond) 2019;80:86-90.

23 Kirk MA, Kelley C, Yankey N et al. A systematic review of the use of the Consolidated Framework for Implementation Research. Implement Sci 2016;11:72.

24 Rycroft-Malone J. The PARIHS framework: a framework for guiding the implementation of evidence-based practice. J Nurs Care Qual 2004;19:297-304.

25 Rycroft-Malone J, McCormack B, Hutchinson AM et al. Realist synthesis: illustrating the method for implementation research. Implement Sci 2012;7:33.
26 Wong G, Westhorp G, Manzano A et al. RAMESES II reporting standards for realist evaluations. BMC Medicine 2016;14:96.

27 Harwood RH, Foss AJE, Osborn F et al. Falls and health status in elderly women following first eye cataract surgery: A randomised controlled trial. $\mathrm{Br}$ J Ophthalmol 2005;89:53-9.

28 Johansen A, Boulton C, Hertz K et al. The National Hip Fracture Database (NHFD) - Using a national clinical audit to raise standards of nursing care. Int J Orthop Trauma Nurs 2017;26:3-6.

29 de Boer AZ, van de Water W, Bastiaannet E et al. Early stage breast cancer treatment and outcome of older patients treated in an oncogeriatric care and a standard care setting: an international comparison. Breast Cancer Res Treat 2020;184:519-26.

30 van de Water W, Bastiaannet E, Egan KM et al. Management of primary metastatic breast cancer in elderly patients-An international comparison of oncogeriatric versus standard care. J Geriatr Oncol 2014:5:252-9.

31 Isaacs B. An introduction to geriatrics. London: Williams \& Wilkins Co, 1965.

32 Gillespie LD, Robertson MC, Gillespie W] et al. Interventions for preventing falls in older people living in the community. Cochrane Database Syst Rev 2009:CD007146.

33 Lord SR, Close JCT. New horizons in falls prevention. Age Ageing 2018:47:492-8.

34 Birks ]. Cholinesterase inhibitors for Alzheimer's disease. Cochrane Database Syst Rev 2006:CD005593.

35 Campbell N, Ayub A, Boustani MA et al. Impact of cholinesterase inhibitors on behavioral and psychological symptoms of Alzheimer's disease: A meta-analysis. Clin Interv Aging 2008;3:719-28.

36 van Leeuwen E, Petrovic M, van Driel ML et al. Withdrawal versus continuation of long-term antipsychotic drug use for behavioural and psychological symptoms in older people with dementia. Cochrane Database Syst Rev 2018;3:CD007726.

37 Livingston G, Sommerlad A, Orgeta V et al. Dementia prevention, intervention, and care. Lancet 2017:390:2673-734.

38 Abraha I, Rimland JM, Trotta FM et al. Systematic review of systematic reviews of non-pharmacological interventions to treat behavioural disturbances in older patients with dementia. the SENATOR-OnTop series. BMJ Open 2017;7:12759.

39 Fitzsimmons PR, Blayney S, Mina-Corkill S et al. Older participants are frequently excluded from Parkinson's disease research. Parkinsonism Relat Disord 2012;18:585-9.

40 Goodman C, Norton C, Buswell M et al. Managing faecal incontinence in people with advanced dementia resident in care homes (FINCH) study: A realist synthesis of the evidence. Health Technol Assess 2017:21:1-229.

41 Fried LP, Tangen CM, Walston ] et al. Frailty in older adults. J Gerontol A Biol Sci Med Sci 2001:56:M146-56.

42 Rockwood K, Mitnitski A. Frailty defined by deficit accumulation and geriatric medicine defined by frailty. Clin Geriatr Med 2011;27:17-26.

43 Clegg A, Bates C, Young ] et al. Development and validation of an electronic frailty index using routine primary care electronic health record data. Age Ageing 2016:45:353-60.

44 Clegg A, Young J, Iliffe S et al. Frailty in elderly people. Lancet 2013;381:752-62

45 Kuh D, Richards M, Cooper R et al. Life course epidemiology, ageing research, and maturing cohort studies: a dynamic combination for understanding healthy ageing. In: Kuh D, Cooper R, Hardy R et al (eds). The Life Course Perspective on Healthy Ageing. Oxford: Oxford University Press, 2014:3-15.

46 Sen P, Shah PP, Nativio R et al. Epigenetic mechanisms of longevity and aging. Cell 2016;166:822-39.

47 Passarino G, de Rango F, Montesanto A. Human longevity: genetics or lifestyle? It takes two to tango. Immun Ageing 2016;13:12.

48 Cruz-Jentoft AJ, Bahat G, Bauer ] et al. Sarcopenia: revised European consensus on definition and diagnosis. Age Ageing 2019:48:16-31. 
49 Cruz-Jentoft AJ, Landi F, Schneider SM et al. Prevalence of and interventions for sarcopenia in ageing adults: A systematic review. Report of the International Sarcopenia Initiative (EWGSOP and IWGS). Age Ageing 2014:43:48-759.

50 Cruz-Jentoft AJ, Sarcopenia Sayer AA.. Lancet 2019;393:2636-46.

51 Dodds RM, Granic A, Robinson SM et al. Sarcopenia, long-term conditions, and multimorbidity: findings from UK Biobank participants. J Cachexia Sarcopenia Muscle 2020;11:62-8.

52 Morley JE. Pharmacologic options for the treatment of sarcopenia. Calcif Tissue Int 2016;98:319-33.

53 Migliavacca E, Tay SKH, Patel HP et al. Mitochondrial oxidative capacity and NAD+ biosynthesis are reduced in human sarcopenia across ethnicities. Nat Commun 2019;10:5808.

54 Cummings JL, Tong G, Ballard C. Treatment combinations for Alzheimer's disease: current and future pharmacotherapy options. J Alzheimers Dis 2019;67:779-94.

55 Atri A. Current and future treatments in Alzheimer's disease. Semin Neurol 2019;39:227-40.

56 Pfistermeister B, Tümena T, Gaßmann KG et al. Anticholinergic burden and cognitive function in a large German cohort of hospitalized geriatric patients. PLOS ONE 2017;12:e0171353.

57 Kirkland JL, Tchkonia T. Senolytic drugs: from discovery to translation. J Intern Med 2020;288:518-36.

58 Cox LS, Bellantuono I, Lord JM et al. Tackling immunosenescence to improve COVID-19 outcomes and vaccine response in older adults. Lancet Healthy Longev 2020;1:e55-7.

59 du Vaure CB, Ravaud P, Baron G et al. Potential workload in applying clinical practice guidelines for patients with chronic conditions and multimorbidity: A systematic analysis. BMJ Open 2016;6:e010119.

60 Piggott KL, Mehta N, Wong CL et al. Using a clinical process map to identify prescribing cascades in your patient. BMJ 2020;368:m261.

61 Whitty CJM. Harveian Oration 2017: Triumphs and challenges in a world shaped by medicine. Clin Med 2017;17:537-44.

62 Abdullah-Koolmees $\mathrm{H}$, van Keulen AM, Nijenhuis M et al. Pharmacogenetics guidelines: overview and comparison of the DPWG, CPIC, CPNDS, and RNPGx guidelines. Front Pharmacol 2020;11:595219.

63 Forester BP, Parikh SV, Weisenbach S et al. Combinatorial pharmacogenomic testing improves outcomes for older adults with depression. Am J Geriatr Psychiatry 2020;28:933-45.

64 McMurdo MET, Witham MD, Gillespie ND. Including older people in clinical research. BMJ 2005;331:1036-7.

65 Lam HR, Chow S, Taylor $\mathrm{K}$ et al. Challenges of conducting research in long-term care facilities: A systematic review. BMC Geriatrics 2018;18:242.
66 Luff R, Laybourne A, Ferreira Z, Meyer ]. A guide to research with care homes. Quality in Ageing and Older Adults 2015;16:186-94.

67 Welch C, Welch C, McCluskey L et al. Growing research in geriatric medicine: A trainee perspective. Age Ageing 2020;49:733-7.

68 Soiza RL, Scicluna C, Thomson EC. Efficacy and safety of COVID-19 vaccines in older people. Age Ageing 2021;50:279-83.

69 Lloyd-Sherlock P, Muljono P, Ebrahim S. Ageism in Indonesia's national covid-19 vaccination programme. BMJ 2021;372:n299.

70 Shrotri M, Krutikov M, Palmer T et al. Vaccine effectiveness of the first dose of ChAdOx1 nCoV-19 and BNT162b2 against SARS-CoV-2 infection in residents of long-term care facilities (VIVALDI study). medRxiv 2021.03.26.21254391.

71 Witham MD, Anderson E, Carroll C et al. Developing a roadmap to improve trial delivery for under-served groups: Results from a UK multi-stakeholder process. Trials 2020;21:694.

72 Witham MD, McMurdo MET. How to get older people included in clinical studies. Drugs Aging 2007;24:187-96.

73 Lunt E, Ong T, Gordon AL et al. The clinical usefulness of muscle mass and strength measures in older people: A systematic review. Age Ageing 2021:50:88-95.

74 Harrison JK, van der Wardt V, Conroy SP et al. New horizons: The management of hypertension in people with dementia. Age Ageing 2016:45:740-6.

75 PROTECT-CH Prophylactic Therapy in Care Homes Trial. PROTECT-CH is a UK-wide clinical trial to identify treatments that can protect care home residents from developing COVID-19. University of Nottingham. www.protect-trial.net [Accessed 30 April 2021].

76 Amaratunga D, Cabrera J, Sargsyan D et al. Uses and opportunities for machine learning in hypertension research. Int ] Cardiol Hypertens 2020;5:100027.

77 House of Lords Science and Technology Select Committee. Ageing: Science, technology and healthy living. House of Lords, 2021. https://publications.parliament.uk/pa/ld5801/ldselect/ Idsctech/183/183.pdf [Accessed 30 April 2021].

Address for correspondence: Prof Adam Gordon, Room 4113, Derby Medical School, Royal Derby Hospital, Uttoxeter Road, Derby DE22 3NE, UK.

Email: adam.gordon@nottingham.ac.uk

Twitter: @adamgordon1978 